\title{
Recherches qualitatives
}

\section{Construire une posture d'altérité pour comprendre des pratiques expertes : l'instruction située au pair comme mode original d'entretien compréhensif}

\section{Cathy Rolland}

Volume 37, numéro 1, été 2018

La recherche qualitative, un univers de nuances

URI : https://id.erudit.org/iderudit/1049456ar

DOI : https://doi.org/10.7202/1049456ar

Aller au sommaire du numéro

Éditeur(s)

Association pour la recherche qualitative (ARQ)

ISSN

1715-8702 (numérique)

Découvrir la revue

Citer cet article

Rolland, C. (2018). Construire une posture d'altérité pour comprendre des pratiques expertes : l'instruction située au pair comme mode original d'entretien compréhensif. Recherches qualitatives, 37(1), 75-95.

https://doi.org/10.7202/1049456ar
Résumé de l'article

Fondée sur une quête de l'altérité, la démarche compréhensive qui anime la recherche anthropologique se développe au coeur des interactions dialogiques entre le chercheur et les enquêtés. Ces interactions s'accompagnent d'une épreuve originale lorsque la culture investiguée est familière au chercheur. La contribution vise le dévoilement de la transformation progressive des relations dialogiques spontanément instituées. Le processus de négociation des postures d'interlocution a impliqué de la chercheuse une activité réflexive sur les modes de communication qu'elle a naturellement contribué à installer au fil de l'enquête. Cette activité lui a permis d'élaborer une conscience de son altérité, propice à l'instauration négociée d'échanges dialogiques, fondés sur la reconnaissance mutuelle entre elle et les entraîneurs d'une asymétrie de connaissances à propos des pratiques étudiées. Les enquêtés ont pu s'enrôler dans une activité d'instruction située, dirigée vers la chercheuse reconnue comme pair, et dévoiler les connaissances implicites, engagées dans leur activité professionnelle. 


\title{
Construire une posture d'altérité pour comprendre des pratiques expertes : l'instruction située au pair comme mode original d'entretien compréhensif
}

Cathy Rolland, maître de conférences

Université Clermont Auvergne, France

\begin{abstract}
Résumé
Fondée sur une quête de l'altérité, la démarche compréhensive qui anime la recherche anthropologique se développe au cœur des interactions dialogiques entre le chercheur et les enquêtés. Ces interactions s'accompagnent d'une épreuve originale lorsque la culture investiguée est familière au chercheur. La contribution vise le dévoilement de la transformation progressive des relations dialogiques spontanément instituées. Le processus de négociation des postures d'interlocution a impliqué de la chercheuse une activité réflexive sur les modes de communication qu'elle a naturellement contribué à installer au fil de l'enquête. Cette activité lui a permis d'élaborer une conscience de son altérité, propice à l'instauration négociée d'échanges dialogiques, fondés sur la reconnaissance mutuelle entre elle et les entraîneurs d'une asymétrie de connaissances à propos des pratiques étudiées. Les enquêtés ont pu s'enrôler dans une activité d'instruction située, dirigée vers la chercheuse reconnue comme pair, et dévoiler les connaissances implicites, engagées dans leur activité professionnelle.

Mots clés

ANTHROPOLOGIE COGNITIVE, ALTÉRITÉ, POSTURES D'INTERLOCUTION, INSTRUCTION SITUÉE AU PAIR
\end{abstract}

\section{Introduction}

Avec pour toile de fond une étude compréhensive des pratiques effectives d'entraîneurs sportifs et des connaissances dont elles témoignent, l'article porte sur les modalités concrètes et originales d'investigation ethnographique : l'expérience de terrain de la chercheuse et les relations humaines qui la constituent. En effet, la pratique de terrain et l'engagement exploratoire des logiques des acteurs qu'elle suppose constituent le pivot de la connaissance anthropologique et de sa démarche inductive. Elle génère une expérience subjective, des autres et de soi-même, susceptible de fournir des preuves empiriques méthodologiquement valables (Copans,

RECHERCHES QUALITATIVES - Vol. 37(1), pp. 75-95.

LA RECHERCHE QUALITATIVE, UN UNIVERS DE NUANCES

ISSN 1715-8702 - http://www.recherche-qualitative.qc.ca/revue/

(C) 2018 Association pour la recherche qualitative 
1999). Dans un régime scientifique qui relève de la plausibilité (plausibilité des matériaux empiriques élaborés en regard des situations investiguées et plausibilités des assertions interprétatives relativement aux matériaux descriptifs), le caractère valide des matériaux, auquel il s'agit de veiller, est conditionné par la rigueur de leur constitution (Olivier de Sardan, 1996). Or, la conquête de l'objectivité scientifique suppose la reconnaissance du mode spécifique d'élaboration de la connaissance anthropologique (Laplantine, 1987) : l'interaction dialogique. Cette connaissance naît bien du terrain, d' « un travail symbolique» de construction de sens (Kilani, 1995, p. 44) entre l'anthropologue et ses informateurs au fil de situations d'interactions, en particulier d'interlocution. Les situations d'énonciation du vécu tel qu'il est parlé par l'Autre', à l'adresse du chercheur, invitent au déploiement d'une pratique compréhensive, car c'est dans l'échange entre interlocuteurs que les messages se négocient et se révèlent. Objectiver cette construction dialogique, ainsi que sa dynamique, suppose du chercheur le développement d'un processus réflexif, qui inclut dans le regard porté sur les pratiques étudiées les relations qu'il a contribué à développer avec les acteurs. La réflexivité, tout en étant une procédure d'objectivation de la recherche, une originalité scientifique propre aux approches inductives en anthropologie (Bordeleau, 2005), apparaît comme une condition de production de la connaissance (Leservoisier, 2005). Elle invite à considérer les phénomènes subjectifs et intersubjectifs « non seulement comme des objets de connaissance scientifique, mais aussi comme des instruments de recherche » (Anadón \& Guillemette, 2007, p. 27). Elle permet d' " analyser le langage de l'altérité tel qu'il se donne dans la communication dialogique » (Affergan, 1987, p. 254), tel qu'il cherche à signifier, à se rendre intelligible pour autrui.

En restituant notre ${ }^{2}$ expérience de terrain, au travers de la description d'épisodes d'interactions vécues au contact des entraîneurs, notre projet est de révéler les processus d'objectivation des phénomènes intersubjectifs à l'œuvre au cours de l'enquête. L'activité réflexive, mise en œuvre au fil de l'étude, visait à saisir les caractéristiques des interactions vécues avec les entraîneurs, dans le but de les ajuster au profit du projet compréhensif. Bien que nourries d'un engagement réflexif constant sur le travail empirique, les régulations in situ des modes d'interaction ont relevé d'un bricolage empirique et expérimental (Morrissette, Demazière, \& Pépin, 2014), car elles ont émergé dans des situations marquées par l'imprévisibilité des évènements et des phénomènes relationnels qui s'y sont instaurés. Ces régulations ont abouti à l'instauration d'une dialogie, fondée sur la reconnaissance mutuelle entre nous et les participants, et d'une asymétrie de compétences concernant des pratiques constitutives d'un champ culturel qui nous est pourtant familier. En effet, nous avons progressivement choisi des situations de recherche dans lesquelles nous avons pu lever les implicites quant aux postures d'interlocution générées par notre statut de chercheuse et formatrice en gymnastique artistique (GA). Au lieu d'être implicitement 
considérée comme une observatrice avertie, nous avons pu faire reconnaître aux entraîneurs notre méconnaissance des savoir-faire qu'ils engageaient dans certaines conditions de pratique. Les espaces discursifs ainsi créés ont caractérisé un mode original d'entretien que nous nommons l'instruction située au pair. Dans celui-ci, les interactions dialogiques ont favorisé l'instauration et le maintien de l'altérité indispensable à l'investigation compréhensive des pratiques étudiées (eu égard aux conditions de familiarité de la chercheuse avec le champ de pratique). Nous montrerons comment les circonstances de sa mise en œuvre, dans la temporalité des situations de travail des entraîneurs, nous ont offert les conditions propices au dévoilement de leurs connaissances professionnelles.

\section{Questionner les pratiques d'intervention d'entraîneurs sportifs}

L'étude empirique présentée visait la compréhension des élaborations cognitives et perceptives que des entraîneurs experts dans la discipline sportive de la GA manifestent au cours de séquences d'enseignement des habiletés gymniques. Il s'agissait de saisir la façon selon laquelle les entraîneurs interviennent auprès des gymnastes pour perfectionner leurs réalisations lorsqu'ils s'exercent sur les agrès ${ }^{3}$; conjointement était visée la caractérisation de leurs perceptions et connaissances des productions corporelles engagées dans l'actualité de ces situations.

\section{Explorer les connaissances implicites impliquées dans l'activité d'entraînement}

Le projet de dévoilement de ces connaissances d'expérience est ancré dans notre biographie d'enseignante d'éducation physique et sportive et d'entraîneure en GA. Bien que diplômée dans ces deux domaines d'intervention, spécialiste de cette activité sportive et nourrie de la culture gymnique en tant que pratiquante, juge et entraîneure, nos compétences à perfectionner des réalisations d'habiletés que nous n'avions préalablement jamais enseignées ont été occasionnellement mises à l'épreuve lors de séances d'entraînement. Engagée de manière inopinée à enseigner de telles habiletés, nous avons fait l'expérience de difficultés à repérer, dans leurs tentatives de réalisation, des éléments défaillants afin d'intervenir de manière pertinente auprès d'elles pour faciliter leurs apprentissages. Ces difficultés vécues nous ont permis de considérer avec une acuité nouvelle le rapport simpliste (Durand, 2002) des connaissances académiques (que nous avions acquises en formation initiale) aux pratiques d'intervention, rapport proposé par le modèle de la science appliquée.

Nos tâtonnements d'enseignante ont aiguisé notre intérêt pour cette intelligence professionnelle, ces « ficelles du métier » qui s'acquièrent dans le corps à corps avec la spécificité d'un milieu (Becker, 2002; Stroobants, 2009). Irréductibles aux savoirs génériques des ouvrages pédagogiques, ces «connaissances tacites" s'inscrivent ontologiquement dans l'expérience corporelle, dans l'engagement sensori-moteur de l'acteur immergé dans des environnements sollicitant son intelligence pratique (Polanyi, 1966). Le projet scientifique s'est donc inscrit dans une épistémologie de 
l'agir professionnel (Schön, 1994). Ce modèle envisage le praticien non plus comme un technicien rationnel qui applique en situation des savoirs formalisés préalablement appris, mais comme un professionnel réflexif qui élabore conjointement, dans la situation problématique qu'il vit, des pratiques adaptatives et des connaissances originales. L'engagement productif de l'entraîneur (optimiser les performances sportives des pratiquants notamment) est considéré générer une activité constructive ${ }^{4}$ (Samurçay \& Rabardel, 2004), c'est-à-dire la production de ressources cognitives ajustées aux problèmes qu'il configure dans l'actualité de son intervention. Selon cette perspective, la cognition, comme objet de recherche, est abordée dans son effectivité comme activité nécessairement dynamique et changeante (Steiner, 2008).

\section{Étudier la cognition en approchant l'expérience professionnelle}

Approcher la rationalité pratique ${ }^{5}$ des entraîneurs, enracinée dans leurs activités ordinaires, supposait d'adopter une théorie de la cognition qui considère le caractère expérientiel des processus cognitifs qui les supportent. Les modélisations de la cognition par les deux paradigmes dominants des sciences cognitives (paradigmes cognitiviste et énactif) sont marquées par une rupture épistémologique inhérente à la lecture qu'un observateur peut effectuer du système cognitif étudié. L'approche cognitiviste traditionnelle, ou paradigme computationnel, appréhende l'activité du système selon une "lecture sémantique » (Peschard, 2004) qui consiste à apprécier, d'un point de vue extérieur au système, la qualité de sa cognition. Les processus cognitifs sont assimilés à des représentations cognitives, équivalents symboliques d'un monde dont les propriétés sont considérées comme prédonnées, préexistantes à l'interaction du sujet avec celui-ci. La fidélité de ces représentations à la réalité objectivée est ainsi susceptible d'être jugée. Elle est approchée par le regard d'un observateur qui se suppose omniscient lorsqu'il lit l'activité.

Quant à elles, les théories énactives proposées par Varela (1989) et Varela, Thompson et Rosch (1993) s'inscrivent dans une ontologie phénoménologique. Le processus cognitif n'est plus modélisé comme la mise en relation de deux pôles prédéterminés que sont le sujet et l'objet objectivé à connaître, mais comme un processus relationnel. Considéré comme autonome ou autoadaptatif (Maturana \& Varela, 1994), ce processus se caractérise par l'émergence, au fil des interactions avec l'environnement, de domaines cognitifs ou relationnels comme autant de zones de perturbations de la structure du système, susceptibles de provoquer sa transformation ainsi que celle du monde qu'il connaît. En situant l'expérience, c'est-à-dire le processus relationnel simultanément coconstitutif du sujet et de son monde phénoménal, comme objet central dans l'étude de la cognition, les théories énactives proposent une «lecture participative» (Peschard, 2004) du système cognitif, qui consiste à prêter attention aux perspectives signifiantes que l'acteur fait émerger dans la dynamique de ses interactions avec les environnements qu'il élabore ou énacte. 
Dans ce cadre théorique choisi pour l'étude, l'analyse des processus cognitifs relève d'un projet descriptif fortement ancré dans le projet de la phénoménologie (Dosse, 1995); il vise l'accès aux phénomènes en tant qu'objets d'expérience singulière pour l'acteur. Il suppose de dépasser le premier niveau de description des comportements observables des entraîneurs (mouvements corporels, paroles, postures, placements spatiaux) dans les contextes matériels et humains (l'activité des gymnastes notamment) d'intervention. La compréhension des processus cognitifs suppose d'éclairer ces comportements des significations qu'ils ont pour les entraîneurs euxmêmes. Elle nécessite ainsi un travail de description de l'activité, dans ses dimensions observable et significative pour eux.

\section{Approche anthropologique et espaces discursifs}

Les positionnements épistémologiques et théoriques adoptés présentent des conséquences majeures: a) l'exigence d'une analyse de l'activité en situation écologique et la construction de méthodes d'investigation permettant de saisir les significations que les entraîneurs assignent aux productions motrices des gymnastes, ainsi qu'à leurs propres interactions avec eux; b) l'impératif de l'adoption par le chercheur d'une attitude critique vis-à-vis des construits sociaux, des élaborations théoriques susceptibles de prescrire les pratiques d'entraînement ou de les expliquer $a$ priori.

L'inscription de l'étude dans le champ disciplinaire de l'anthropologie cognitive a offert un cadre paradigmatique respectueux de ces exigences épistémologiques. En effet, l'anthropologie interprétative (Geertz, 1973), en filiation à une anthropologie pragmatique (Kant, 1797/1964), porte son regard vers l'action dans ses procédures signifiantes pour l'acteur, sa rationalité expérientielle, sans lui imposer des structures explicatives préétablies. La dynamique compréhensive qui l'anime est ancrée sur une quête de l'altérité, sur «l'invention de l'autre ${ }^{6} »$ (Kilani, 1995), reconnu comme sujet « actant», capable d'expliciter sa propre subjectivité. Cet « objet» anthropologique se dessine progressivement dans la confrontation entre subjectivités culturelles et personnelles (Caratini, 2004), celle du chercheur et celle des acteurs étudiés, avec leur originalité socioculturelle, leur histoire. Saisir cette altérité suppose du chercheur qu'il l'envisage comme « inanticipable, à savoir comme non prédicable par un concept a priori » (Affergan, 1987, pp. 267-268). L'ancrage anthropologique invite ainsi à

rendre compte d'actions, d'activités et d'interactions, [tel] qu'elles se font, [à se démarquer] des discours officiels que les professionnels tiennent sur elles-mêmes, de leurs codifications théoriques, juridiques et administratives, des justifications que les praticiens donnent de leurs actions (Cefaï, 2010, p. 40).

Conjointement, l'ethnologue doit s'efforcer d'expliciter son rapport à la culture investiguée afin d'éviter l'écueil de l'ethnocentrisme. L'enjeu est la compréhension, au 
moyen d'une expérience singulière, des phénomènes humains tels que les acteurs les vivent (Laplantine, 1987). Dans ce mélange subtil de familiarité et de distance, le véritable défi du chercheur réside, pour Latour et Woolgar (1988), dans la conquête d'une posture de recherche distanciée. Cette conquête apparaît d'autant plus sensible lorsqu'indigène parmi les indigènes, l'ethnologue se confronte à un univers culturel qui lui est coutumier.

Créer des conditions originales d'observation participante : construire l'altérité

Nous étions engagée dans une ethnologie de proximité ou «endo-ethnologie» (Ouattara, 2004). En réduisant l'écart ethnoculturel entre les enquêtés et le chercheur, elle court-circuite la clause ordinaire d'exotisme (Urbain, 2003) et contraint à approfondir la question du type d'éclairage auquel il est possible de soumettre les pratiques étudiées (Kilani, 1992). Un «jeu de distanciation intellectuelle», comme « attitude épistémologique réversible » (Agier, 2004, p. 106), potentialise l'accès à l'altérité. Il suppose l'adoption d'une posture réflexive sur les conditions d'interactions dans lesquelles les connaissances se développent. Ce défi s'est imposé à nous, comme un postulat cognitif et un impératif méthodologique, pour investir des pratiques familières. Il nous a offert les moyens de créer des ajustements interactifs originaux, favorables à l'élaboration progressive d'une connaissance de l'activité étudiée.

\section{Les terrains de l'enquête}

L'enquête de terrain, d'une durée de trois ans, s'est appuyée sur la participation de quatorze entraîneurs (deux femmes et douze hommes) qui intervenaient dans leur structure d'entraînement respective, en tant que professionnels expérimentés. Ils présentaient une expérience, en tant qu'entraîneurs, d'au moins huit ans et étaient reconnus par la communauté de la GA comme des experts (car nommés ainsi et placés, pour certains, à des postes sensibles au sein de la fédération). En tant que représentants d'une culture de l'entraînement, «qualifiable par un langage, des croyances et des théories implicites, ainsi que des catégories de description des actions sportives et des procédures d'entraînement » (Durand, Hauw, Leblanc, Saury, \& Sève, 2005, p. 4), ils ont constitué une unité sociale cohérente offrant à notre analyse par cas (Passeron \& Revel, 2005) des descriptions multiples et contrastées.

Trois terrains différents ont été examinés. L'enquête a débuté dans un club féminin de niveau national avec deux entraîneurs participants, avec lesquels nous avions précédemment partagé une activité d'entraînement. Cet engagement initial dans un univers familier a facilité l'acceptabilité de notre présence, tout en nous contraignant à opérer une rupture dans les postures habituelles, pour fonder des échanges praticien-chercheuse. L'enquête s'est poursuivie, six mois plus tard, au sein de deux Pôles France ${ }^{7}$ masculin de la Fédération française de gymnastique avec douze entraîneurs. 
Le choix d'élargir le terrain d'investigation à la gymnastique masculine nous a permis d'assister à des pratiques de perfectionnement d'habiletés que nous n'avions jamais enseignées (car spécifiques de la pratique masculine) et dont l'observation était susceptible de générer chez nous un étonnement favorable à un engagement dans une activité compréhensive. Ce changement de terrain nous a offert des conditions favorables à la négociation avec les entraîneurs d'espaces discursifs propices à l'enquête.

\section{L'entretien d'explicitation comme entretien compréhensif}

L'accès aux significations incarnées, qui accompagnent de manière muette l'activité, suppose la verbalisation par l'acteur des actes qui la composent, en dehors du temps de leur accomplissement ${ }^{8}$. En cohérence avec le paradigme énactif, diverses méthodologies de verbalisation (autoconfrontation simple ou croisée, entretien d'explicitation, instruction au sosie, etc.) aspirent à encourager une description de l'expérience telle qu'elle a été vécue. Toutes visent à accompagner l'accès rétrospectif à l'expérience passée malgré un usage des traces, un engagement du chercheur et des finalités qui divergent. Pour notre étude, nous avons, dans un premier temps, organisé des entretiens d'explicitation (Vermersch, 1994), dans un délai très court après l'entraînement ou une séquence d'enseignement. Nous avons utilisé les matériaux d'observation du flux de comportements des entraîneurs, dans leur contexte d'émergence (leurs communications verbales, leurs placements et déplacements, leurs gestes, leurs manipulations corporelles des gymnastes), comme traces de l'activité pour provoquer et soutenir une activité d'explicitation de leurs actes. L'entraîneur était invité à décrire ce qu'il repérait comme particulièrement significatif dans les réalisations motrices, à préciser ce qu'il percevait et les liens avec les prescriptions de transformation qu'il proposait aux gymnastes. Ces entretiens visaient la construction de descriptions en seconde personne, qui relèvent de ce que le chercheur peut comprendre, ressentir avec empathie, de l'expérience subjective passée de l'entraîneur (Vermersch, 1999).

Une seconde forme d'organisation temporelle d'échanges s'est progressivement installée au cours de l'enquête dans les Pôles France. Elle s'est caractérisée par ce que Filliettaz (2011) nomme des «explicitations situées », c'est-à-dire une production discursive originale, car adressée par le praticien au chercheur de manière spontanée et située dans le déroulé de son activité. Nous avons alors pu observer un engagement alterné de l'entraîneur dans le guidage de l'activité d'apprentissage des gymnastes et dans la production d'un discours professionnel destiné à nous permettre de comprendre son activité auprès d'eux, non a posteriori de la séquence d'activité d'enseignement, mais directement durant celle-ci. Cette modalité d'entretien d'explicitation est originale à double titre : a) elle s'inscrit dans les conditions spatiales et temporelles du déroulement de l'activité, les temps d'explicitation s'intercalant entre les temps 
d'intervention; b) elle se caractérise par un espace dialogique progressivement élaboré dans la dynamique de l'enquête de terrain, à la fois de manière volontaire et opportune. En effet, les circonstances de l'enquête nous ont amenée à considérer et à réfléchir la particularité des situations d'interlocution, et à dépasser le cadre des relations interpersonnelles préconisé par Vermersch (1994). Ce cadre consiste à demander aux participants un accord de principe et à adopter une posture neutre pour conduire l'acteur vers une position d'évocation, dans laquelle la situation passée est davantage présente pour lui que la situation actuelle d'interlocution.

Pour mieux saisir l'originalité des situations dialogiques, nous nous sommes astreinte à nous penser nous-mêmes, en tant que "principal médium de l'enquête " (Cefaï, 2010, p. 7), comme partie intégrante d'espaces dialogiques qui « épouse[nt] les formes du langage ordinaire »(Althabe, 1990, p. 1), bien que sous-tendus par des enjeux scientifiques.

\section{De l'interaction silencieuse aux balbutiements de l'interaction discursive}

Les conditions d'interactions dialogiques ne pouvant être ni imposées ni déterminées a priori, il fallait nous engager dans l'univers des salles d'entraînement, amorcer des relations avec les entraîneurs afin d'espérer pouvoir les infléchir, les orienter au bénéfice de l'enquête.

\section{De l'observateur effacé à l'observateur inséré dans la dynamique des pratiques}

L'enquête s'est organisée entre distance et proximité avec l'espace physique et social créé par les entraîneurs en activité. Contrairement au premier terrain où nous avions précédemment noué des relations en tant qu'entraîneure, et avons aisément trouvé une place dite de chercheuse auprès des entraîneurs en activité, le deuxième terrain d'étude suivant a été l'occasion d'une expérience nous contraignant à réfléchir à la construction de notre place d'ethnologue. Cette place s'est dessinée conjointement à l'investissement progressif de l'espace physique de la salle d'entraînement. En effet, à notre arrivée, confinée dans un espace dédié aux observateurs de passage, nous avons éprouvé des difficultés à identifier les gymnastes dont les entraîneurs avaient la responsabilité. Ces derniers intervenaient auprès de gymnastes, disséminés sur les agrès, parfois de loin par des injonctions verbales courtes, parfois en créant une relation de proximité lors de manipulations corporelles des gymnastes, d'explications verbales plus fournies. Nous étions incapable de repérer ce que les entraîneurs, qui se déplaçaient souvent, observaient, et de lier leurs interventions à la réalisation motrice qu'ils avaient vue. L'espace d'entraînement, vaste, nous rendait souvent leurs adresses verbales inaudibles. Il a fallu nous extirper de cette position spatiale, nous engager dans l'espace de travail et construire notre place dans l'espace social.

L'enjeu consistait à pouvoir « voir comme » les entraîneurs, c'est-à-dire porter le regard sur le gymnaste qu'ils étaient en train d'observer, et pouvoir entendre, c'està-dire pouvoir «mettre l'oreille à portée des énonciations en cours ou probables » 
(Copans, 1999, p. 83), et ce, tout en maintenant une distance propice à l'exercice de leur activité. Des séquences d'intervention, circonscrites dans un espace physique plus restreint (autour d'un agrès ou d'un atelier), ont été l'occasion de nous rapprocher physiquement des protagonistes et d'amorcer une observation de proximité. Un espace social s'est ainsi créé, potentialisateur d'interactions dialogiques, qui restaient à construire.

\section{D'interactions silencieuses à l'initiation d'échanges collaboratifs}

Dans les trois terrains de recherche, nos premières expériences se sont caractérisées par une solitude particulière, marquée par un silence déroutant. Ce dernier, installé entre le chercheur et les entraîneurs, a été saisi comme possédant un "réel statut discursif» (Arshad, 2000, p. 2). Sa compréhension s'est inscrite entre les deux extrémités des interactions humaines qu'il représente selon Arshad (2000), à savoir « communication danger » et « communication fusion/communion».

Le silence comme signe d'une proximité culturelle

Les interactions silencieuses mettaient en relief notre difficulté à entamer une communication. L'investigation de notre premier terrain s'est accompagnée de l'impression d'être emportée par le cours de l'entraînement sans pouvoir nous y extraire pour y porter un regard questionnant. Nous observions les interventions des entraîneurs comme anesthésiée, car rien, dans les réalisations des gymnastes et les comportements des entraîneurs, ne venait troubler notre relative quiétude; aucun fait étrange ne venait rallumer notre curiosité. Ces expériences ont révélé, au fil de notre activité réflexive, notre lien d'appartenance à la société étudiée, notre niveau de membership (Amiel, 2004), lien suffisamment dense pour que nous adhérions sans réserve au sens commun des enquêtés, sens de leurs propos et gestes professionnels. Cette connivence avec les pratiques observées ensevelissait le sens, les signes et les significations sous le familier (Urbain, 2003). Elle s'exprimait dans la perception implicite d'une adéquation entre les productions des gymnastes (avec leurs erreurs, leurs manques ou leurs qualités) et les interventions des entraîneurs auprès d'eux (conseils, remarques, etc.). Nos approbations tacites à l'activité en cours nous conduisaient à rester silencieuse ou à formuler mécaniquement des questions similaires pour approcher l'expérience perceptive des entraîneurs telles que « qu'est-ce que tu as repéré? », « comment l'as-tu perçu? ». Nos questions engendraient des échanges qui ne révélaient pas un engagement compréhensif nourri d'une curiosité véritablement piquée : nous nous contentions le plus souvent des réponses spontanées des entraîneurs et n'enquêtions pas sur les liens entre les indices perceptifs que les entraîneurs indiquaient et les recommandations qu'ils délivraient aux gymnastes. L'analyse d'un extrait d'échanges avec l'entraîneure B est révélatrice d'un partage de sens avec elle, en particulier de catégories perceptives, qui engendre une description sommaire de sa part. Après avoir indiqué à une gymnaste qu'elle "avait la poitrine un peu sortie » 
durant la réalisation d'un saut appelé lune (à l'agrès saut), l'entraîneure $\mathrm{B}$ répond à notre question « comment tu l'as vu? » :

Je regarde le préappel, quand elle monte les bras, tu le vois... et après je le confirme sur le saut mais ce n'est pas une déduction parce que je l'ai vu avant sur le tremplin... Sur le saut, je le vois à la tête qui sort quand elle est en appui les mains sur le saut (Entraîneure B).

L'entraîneure B n'est plus alors invitée à clarifier le sens de l'expression poitrine sortie, pas plus que le lien qu'elle fait entre les positions de la tête et la poitrine.

Réfléchir cette communion dialogique qui s'exprimait dans des silences ou des échanges discursifs superficiels a permis d'identifier notre défi : se défamiliariser de notre propre regard afin de susciter à nouveau en nous la perspicacité de la vision de son monde par l'Autre (Urbain, 2003). Nous l'avons relevé en examinant des pratiques d'entraînement en gymnastique masculine.

\section{Le silence comme signe d'une violence symbolique ressentie}

Nous avons fait l'expérience, au sein des Pôles France, d'un silence d'une autre nature, celui-ci s'intercalant entre les échanges verbaux que nous avions commencés. Les collaborations polies de début d'enquête reposaient sur une configuration de rôles dans laquelle la chercheuse déclenchait la discussion et orientait les échanges, les entraîneurs s'efforçant de répondre aux questions posées. Sollicités, les entraîneurs coopéraient volontiers et étaient attentifs à expliciter leur activité. Cependant, la méfiance et la gêne qui transparaissaient parfois de la brièveté de leurs réponses, ainsi que de quelques comportements d'évitement, exigeaient de notre part une réflexion sur les significations qu'ils assignaient à la situation d'interlocution créée. Plutôt coutumiers de la présence de chercheurs au sein des Pôles, ils l'étaient moins des méthodologies d'entretien que nous avions mobilisées, ainsi que de la finalité compréhensive annoncée. Cette dernière n'était pas en cohérence avec nos questionnements limités, parfois sans demande d'approfondissement. Leurs silences révélaient une violence symbolique ressentie, liée à la crainte manifeste de voir leurs pratiques jugées, voire disqualifiées à partir de catégories surplombantes.

Le caractère artificiel de la relation sociale, où le dialogue est institué par le chercheur à des fins de connaissance (Beaud \& Weber, 2003) est à l'origine, selon Bourdieu, d'une « violence symbolique » (1993, p. 1393) potentiellement ressentie par les enquêtés. Cette violence émane, selon lui, d'une dissymétrie sociale présente chaque fois que l'enquêteur occupe une position supérieure aux enquêtés, dans la hiérarchie des différentes espèces de capital, notamment le capital culturel. Cette hiérarchisation des individus, à partir de l'appréciation plus ou moins explicite de leur capital culturel, apparaît prégnante dans les milieux de l'enseignement et de l'entraînement sportif, fortement imprégnés d'une conception de la science appliquée, qui a orienté les formations dispensées aussi bien au sein des institutions universitaires 
que fédérales. Connue en tant que formatrice d'intervenants dans le domaine des activités physiques et sportives, nous incarnions probablement pour les entraîneurs cette conception d'une pratique d'intervention à construire « en parfaite cohérence avec les références scientifiques » (Piard, 1992, p. 98). Nos regards et nos questionnements étaient ainsi rapidement assimilables à ceux d'un contrôleur averti.

Ces interactions silencieuses, inconfortables pour les deux interactants, mettaient en évidence l'inertie épistémique de notre démarche, tout en constituant une occasion de penser et d'infléchir le délicat dosage entre altérité et identité, différence et unicité. Les entraîneurs devaient être invités à expliciter leur pratique sans ambiguïté avec une forme de demande de justifications théoriques. Afin de lever toute équivoque dans le discours attendu des entraîneurs, nous avons insisté sur notre projet d'étudier leurs connaissances d'expérience. Cependant, l'expérience de terrain a révélé l'insuffisance de ces explications. Les intentions annoncées devaient se concrétiser au sein même des relations dialogiques. Le nouveau terrain de la gymnastique masculine, moins familier pour nous, n'était pas en lui-même porteur de l'altérité convoitée, mais nous a offert les possibilités de sa coconstruction en instaurant progressivement, au sein des interactions, les conditions de réciprocité qui la fonde: saisir autrui et, selon l'expression de Ricœur, (1990), soi-même comme un Autre (Affergan, 1987).

\section{De la connivence intellectuelle à l'asymétrie cognitive}

C'est au cœur de la dynamique des échanges avec les entraîneurs que s'est installé un mode de communication propice à l'élaboration de la connaissance anthropologique. Althabe rappelle en effet que l'investigation ethnologique se développe dans la nonséparation avec la communication ordinaire: l'enquête "épouse les formes du dialogue ordinaire et c'est dans ce dialogue que le chercheur introduit une distance qu'il est condamné à reproduire lors de chaque rencontre » $(1990$, p. 1).

\section{Un espace d'échanges informatifs}

L'investigation des pratiques d'entraînement dans les Pôles France a été aisée du fait que nous possédions la compétence minimale requise pour ce faire, laquelle consiste selon Amiel (2004) à pouvoir conduire un entretien de façon telle que le répondant n'ait pas l'impression désagréable de parler à un martien. Au début de nos interactions avec les entraîneurs, notre ignorance de la culture gymnique a été soupçonnée. Leurs explicitations se restreignaient parfois à des clarifications sémantiques générales. Par exemple, l'entraîneure $\mathrm{D}$, questionnée sur une prescription qu'elle avait adressée à un gymnaste évoluant à la barre fixe : «pousse ta lune», a limité sa réponse à la description de l'habileté nommée lune, sans aborder la transformation qu'elle visait: «[...] la lune, c'est l'élément. C'est un grand tour en avant, avec une prise de mains particulière sur la barre. »

Notre méconnaissance supposée nous plaçait insidieusement dans une position délicate : celle d'une chercheuse sollicitant un professionnel et risquant de lui faire 
perdre un temps précieux pour glaner des connaissances manifestement superficielles. Nous avons alors ressenti la nécessité d'établir un dialogue qui prendrait appui sur le partage d'une connaissance technique et culturelle de la gymnastique. Pour cela, nous avons volontairement distillé, au fil des échanges, des expressions idiomatiques afin de révéler notre statut de membre de la communauté. Le langage indigène, propre à la culture gymnique, concerne en particulier un langage corporel complexe. Les mouvements gymniques sont acrobatiques, car ils engagent le corps dans la production de formes corporelles extraordinaires. Notre compétence à identifier ces mouvements (ou habiletés) dans la réalisation d'un enchaînement, en termes de début et de fin, de combinaisons de rotations transversales et longitudinales, d'orientations corporelles et de relations aux agrès, à nommer ces habiletés, certaines phases de celles-ci (prise d'élan, appel, réception, envol, etc.) ou encore certaines actions techniques (impulsion, antépulsion, etc.), nous est apparue comme une condition nécessaire pour mobiliser notre attention, conjointement sur la production motrice du gymnaste observé et sur l'activité de l'entraîneur pour la transformer. Par ailleurs, notre culture professionnelle nous permettait d'accorder aux scènes observées une consistance, une intelligibilité spontanée qui pouvaient alors être interrogées avec l'aide des entraîneurs. Nous comprenions, par exemple, que l'entraîneur montrait un geste défectueux ou attendu, et il importait alors de recueillir des explicitations relatives à celui-ci.

Nous avons ainsi, au cours des échanges, "convoqué une interconnaissance commune » (Papinot, 2014, p. 40) qui relevait de discours professionnels naviguant entre savoirs formalisés (sans toutefois convoquer des savoirs scientifiques susceptibles de créer une posture surplombante et inquiétante) et langage indigène, voire jargon de spécialiste. Nous avons toutefois veillé à ne pas nous y enfermer, en distinguant le plus lucidement possible les moments où nous étions dans la participation (aide à l'installation du matériel, discussions informelles, etc.) et les moments où nous souhaitions valoriser une posture de chercheuse distanciée. Peu à peu s'est instaurée une relation de confiance fondée sur une charité rationnelle mutuelle (Biache, 2002), sur la reconnaissance réciproque des compétences et connaissances de l'interlocuteur: d'une part, la chercheuse était reconnue compétente à se rendre intelligible l'activité de l'entraîneur ainsi que les habiletés à acquérir; d'autre part, l'entraîneur était investi d'une compétence à enseigner, c'est-à-dire à se rendre intelligible l'activité des gymnastes pour la transformer. Cette reconnaissance s'est accompagnée de l'émergence d'une relation asymétrique entre un expert et une novice dans l'enseignement d'habiletés destiné à préparer une pratique de haut niveau.

Porter notre attention aux pratiques d'entraînement en gymnastique masculine a ainsi généré une perturbation de notre système de référence et a rendu possible l'expérience de l'altérité. L'Autre a pu se révéler au sein même de la relation qu'ont établie les interlocuteurs. 


\section{Un espace d'échanges instructifs}

Les échanges, d'abord fondés sur une logique informative, ont peu à peu évolué vers une logique instructive, caractérisée par une dialogie d'enseignement-apprentissage.

\section{Asseoir une dialogie d'enseignement-apprentissage}

L'asymétrie naissante s'est affirmée avec l'instauration d'un nouveau mode d'interlocution qui a émergé au fil des entretiens. En effet, certains silences de notre part, à la suite des réponses des entraîneurs, ont pris pour eux une signification particulière : non pas, comme nous, comme une incapacité à relancer l'entretien, mais comme une invitation à réfléchir davantage leur activité perceptive passée, leurs intentions, à mieux se faire comprendre. Précisant leurs propos, en proposant par exemple de dessiner ce qu'ils avaient vu, ils ont créé un registre conversationnel dans lequel nous étions placée comme novice à instruire. L'attention portée aux dynamiques sociales des entretiens nous a permis de nous saisir de cette configuration discursive pour l'instaurer de manière plus méthodique. Nous étions initialement présente pour réceptionner les informations sollicitées, dans une conception de l'entretien comme «extraction minière d'informations » (Olivier de Sardan, 1995), et nous étions désormais invitée à accompagner la réflexion des entraîneurs sur leur activité, à asseoir une interaction dialogique dans laquelle pouvait authentiquement se déployer une activité compréhensive. Les configurations d'activités observées nous offrant des îlots énigmatiques, il nous restait à nous engager véritablement à les résoudre, in situ. La relation dialogique a alors été pensée comme une relation d'instruction au cours de laquelle l'entraîneur adressait son discours à un pair, novice dans la transmission des habiletés concernées, pour l'instruire. Nous l'avons investie en visant un engagement soutenu dans un processus d'apprentissage conscient. Conjointement, les entraîneurs perdaient leur rôle d'informateurs au profit de celui de formateurs. Cette modalité dialogique entre un praticien expérimenté et un praticien novice dans le domaine de pratique investigué a permis de créer des conditions propices à l'émergence d'un rapport d'intelligibilité mutuelle. L'entraîneur, reconnu comme praticien réflexif (Schön, 1994), était amené à retrouver le chemin de son expertise (Tochon, 1993), à l'envisager différemment sous le jour des questionnements de la chercheuse.

Lors d'une séquence d'enseignement d'une sortie ${ }^{10}$ aux barres parallèles, notre activité d'apprentissage a bénéficié conjointement des explicitations que l'entraîneure $\mathrm{D}$ nous adressait, des adresses verbales dirigées vers les gymnastes ainsi que de notre engagement corporel dans la situation de travail. Nous étions placée en retrait derrière l'entraîneure en action afin de bénéficier du même angle de vue que le sien sur les gymnastes en mouvement (de profil). Elle manipulait le corps des gymnastes afin d'associer à ses guidages verbaux un guidage corporel et améliorer la réalisation d'une séquence discrète constitutive de l'habileté (Photographie 1). 


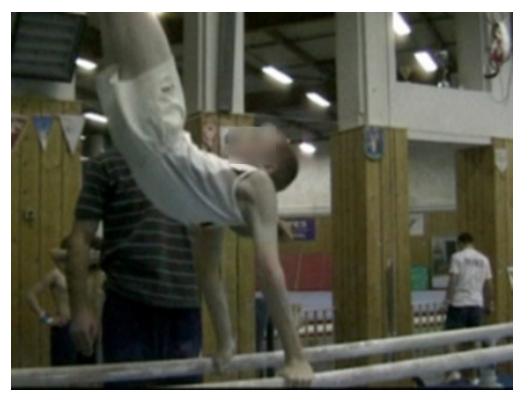

Photographie 1. Séquence discrète ou phase de placement de l'habileté enseignée.

- Entraîneure D au gymnaste : «Il faut maintenir le placement longtemps jusqu'à l'oblique avant de lâcher et là la tête ne finit pas au bout du tapis. »

- Entraîneure D à la chercheuse : «Il doit retomber à l'aplomb de la barre. Là, tu vois, il arrive en avant parce qu'il perd l'appui et ne peut pas poursuivre son placement. »

Elle montre le placement avec sa main et son avant-bras en représentant une ligne positionnée à l'oblique par rapport à l'horizontale des barres. Nous comprenons que le gymnaste se réceptionne en avant (à la fin de la réalisation de l'habileté) par rapport à son appui des mains sur la barre, ce que nous confirmons in situ par l'observation de la position du gymnaste dans la partie avant du tapis.

- Chercheuse à l'entraîneur D : «Qu'est-ce que c'est perdre l'appui? Tu as vu quoi?»

- Entraîneure D à la chercheuse : «On le voit qu'il perd l'appui parce que ses épaules partent en avant; elles ne sont plus à la verticale des mains et il ne peut plus appuyer longtemps sur la barre pour faire monter le bassin. »

\section{Une expérience corporelle fondamentale}

Le maintien dans un processus actif d'apprentissage a consisté à rapporter l'instruction attendue à des moments d'intervention bien spécifiés et contextualisés (c'est-à-dire ses actions associées à celles des gymnastes); il permettait une centration sur la dimension procédurale de l'action vécue. Le déroulement des entretiens, dans l'espace-temps de la pratique d'intervention auprès des gymnastes, a favorisé le maintien du lien agirdiscourir sur l'action. L'apprentissage des pratiques des entraîneurs était ainsi centré sur les conditions originales d'intervention et permettait d'approcher les connaissances qui s'y exprimaient. Nous nous assurions du développement progressif de nos compétences à potentiellement guider les apprentissages des gymnastes. Pour ce faire, nous nous attachions à suivre précisément l'activité de l'entraîneur et ses explicitations afférentes, dans son déroulé temporel au sein d'une séquence d'intervention. C'est ce 
suivi, pas à pas, qui nous a permis d'effectuer une expérience initiatique fondamentale. Au cours de l'enseignement de la même sortie aux barres parallèles, l'entraîneure D explicitait qu'elle observait le «placement du bassin » du gymnaste relativement aux barres durant la phase d'appui avant le lâcher des barres : « Je regarde le bassin, c'està-dire l'alignement et la hauteur; il faut rechercher l'alignement du corps avec le bassin haut, puis renverser en enroulant les jambes sur le buste.» Instruite par cette indication, nous dirigions notre regard en conséquence sur le bassin du gymnaste lors de sa seconde tentative. Surprise par la prescription adressée par l'entraîneure au gymnaste, puisqu'elle portait sur «l'action d'appui» des bras sur les barres et que, focalisée sur le bassin, nous n'avions pas observé d'autres entités corporelles, nous avons demandé à l'entraîneure de nous éclairer sur ce qu'elle avait alors observé. Le processus d'explicitation engagé a permis de mettre à jour que l'entraîneure ne regardait pas spécifiquement le bassin, mais une forme globale plus complexe et dynamique dans laquelle le bassin constituait un des points remarquables : « Je vois le tout, le bassin, les épaules avec l'appui, l'enroulement des jambes. » Le phénomène qui nous est alors apparu, au cours des observations ultérieures des réalisations, est une forme synthétique dessinée par le corps à un moment particulier du mouvement et non plus des entités corporelles isolées. D'abord perçu comme morcelé, le corps en mouvement s'est constitué comme une structure articulée, se configurant dans la temporalité du mouvement en des formes géométriques successives. L'expérience perceptive que nous avons vécue comme «saut qualitatif» (Zeitler, 2003), car apparentée à «l'accès à un nouvel ici, lequel fait voir l'initial comme un autre » (Ouellet, 1992, p. 203), a constitué une véritable donation de sens. En effet, la configuration perceptive de la forme corporelle dynamique a permis celle de saillances perceptives qui ont pris simultanément, en lien avec les transformations visées, une signification cohérente; dans le cas de la sortie aux barres parallèles, il s'agissait du placement des épaules, de la hauteur et de l'enroulement du bassin. L'expérience perceptive de cette forme, découpée dans le plan sagittal (Photographie 1), a permis une avancée considérable dans le travail interprétatif. En nous encourageant à vivre une expérience sensible proche de la leur, les entraîneurs nous ont permis d'élaborer un modèle de compréhension de leur intelligibilité située des réalisations gymniques. Les configurations corporelles, qu'ils nomment phases de placement et qui structurent pour eux les habiletés, constituent des ressources à l'action; en agrégeant de manière cohérente et synthétique les différentes propriétés qu'ils leur assignent dans la situation originale d'intervention, ils créent des voies de régulation diverses, qu'ils expérimentent auprès des gymnastes. 


\section{Conclusion}

La quête de l'altérité, indispensable à notre investigation en anthropologie cognitive, a révélé la corporéité comme clefs de compréhension (Hert, 2014) des phénomènes à l'œuvre dans les situations de terrain que nous avons investies.

Tout d'abord, nos expériences corporelles des situations d'interlocution ont constitué un levier de compréhension de nos interactions avec les entraîneurs. Celles-ci ont révélé comment l'enquêté signifiait à son interlocuteur dans l'intention de se rendre intelligible envers lui (Affergan, 1987). En effet, les processus réflexifs mis en œuvre au fil de l'enquête se sont avérés pratiques, incarnés dans des configurations praticosensibles d'activités et d'interactions (Cefaï, 2010). Nourris d'une attention portée à nos affects, à nos sensations, ils nous ont permis, sur le vif, de rectifier notre façon d'interagir et de maintenir un engagement dans la situation d'enquête. Nous avons ainsi développé peu à peu une conscience de l'altérité dont nous étions porteuse (Althabe \& Hernandez, 2004) au sein des situations dialogiques. En favorisant l'élaboration d'interactions favorables à l'enquête, la réflexivité a pris une dimension «tactique » (Céfaï, 2010, p. 563). Elle a permis aux protagonistes, dans le temps du dialogue, de se reconnaître mutuellement un pan d'asymétrie culturelle les engageant dans des activités conjointes d'enseignement-apprentissage et propices au dévoilement des connaissances d'expérience recherchées. Ces coajustements en situation ont révélé les configurations dialogiques originales comme espaces d'édification des significations de l'agir. L'instruction au pair, par le mode d'interactions dialogiques qu'elle instaure, invite à une reconfiguration de l'activité passée à la lumière de la problématique d'enseignement-apprentissage de la situation actuelle d'interlocution. Elle ne génère pas une ressuscitation de l'expérience passée inscrivant la description dans une ontologie du "Même», mais produit une description sous le «signe de l'Analogue » (Ricœur, 1985, p. 205), dans laquelle l'expérience passée est reconfigurée dans la situation actuelle d'entretien, nourrie de l'activité compréhensive de la chercheuse ${ }^{11}$, de ses interrogations et difficultés d'apprentissage. Sa mise en œuvre située, objectivée par les traces observables de l'activité, a évité l'écueil de l'élaboration d'un discours généralisant, approchant les formes académiques de l'enseignement.

Enfin, notre engagement corporel a constitué une clef de compréhension des connaissances-en-acte, incarnées, des entraîneurs. Dans le paradigme énactif, la cognition comme action incarnée dépend des types d'expériences qui découlent du fait d'être un corps doté de capacités sensorimotrices qui s'inscrivent elles-mêmes dans un contexte plus large, biologique, psychologique et culturel (Varela et al. 1993). La mise en œuvre située de l'instruction au pair nous a permis de faire l'expérience, en immersion, des pratiques étudiées. Ces expériences ont permis de tisser des liens compréhensifs entre les explicitations de l'acteur et sa pratique effective, dans son 
déroulé chronologique. Elles nous ont offert la possibilité d'éprouver corporellement des situations multiples originales et de saisir le caractère ajusté des connaissances aux circonstances locales d'intervention. Dans une logique de continuité entre les instruments de compréhension et les résultats de l'étude, et en cohérence avec notre projet de saisir les processus cognitifs engagés dans des situations écologiques, le modèle théorique que nous avons pu élaborer (Rolland \& Cizeron, 2015) s'appuie sur des matériaux qui mêlent " perçu et exprimé, sensible et intelligible, vécu intime et expression de formes culturelles » (Hert, 2014, p. 30).

\section{Notes}

${ }^{1}$ Affergan (1987) distingue l'autre, avec une minuscule, qui désigne l'individu particulier qui est nommé (untel, là, appartenant à telle culture), de l'Autre, avec une majuscule, qui renvoie à l'universalité du concept, genre ou espèce, et désigne tout ce qui n'est pas soi, extraterritorialisé, délimité par le sujet qui parle. L'autre avec une minuscule est utilisé par d'autres auteurs tels que Kilani (1995) dans une acception similaire du terme Autre tel que déterminée par Affergan.

${ }^{2}$ Pour effectuer le récit de notre expérience de terrain, nous avons choisi de faire usage du nous afin de rendre compte de la recherche d'une posture distanciée. Ce Nous représente le « chercheur et son double » (Cohen, 2002), le chercheur qui, par une analyse réflexive, cherche à mettre à jour ses relations intimes de sujet individuel à l'objet étudié.

${ }^{3}$ Les agrès gymniques sont les structures matérielles sur lesquelles évoluent les gymnastes pour y réaliser des figures corporelles (par exemple les barres parallèles, les anneaux et le cheval d'arçons pour les gymnastes masculins).

${ }^{4}$ L'activité professionnelle peut être envisagée selon deux aspects indissociables (Pastré, 2011) ou coémergents : les aspects productif et constructif (selon la distinction initiée par Samurçay \& Rabardel, 2004), qui renvoient respectivement à la transformation du réel et à la transformation de l'acteur lui-même.

${ }^{5} \mathrm{La}$ rationalité pratique est une rationalité dont la pertinence est opératoire pour l'activité engagée. Elle est non discursive au sens où elle renvoie à des actes comme significations incarnées qui appartiennent au registre d'une conscience "préréfléchie » (Vermersch, 1994), c'est-à-dire une conscience implicite, opaque et syncrétique.

${ }^{6}$ Cet autre nommé par Kilani (1995) renvoie à ce qu'Affergan (1987) désigne par Autre.

${ }^{7}$ Ces pôles sont des structures nationales qui ont pour mission la formation des sportifs de haut niveau.

8 L'activité s'accompagne d'une expérience préréfléchie, vécue sans être immédiatement accessible à la conscience et à la description verbale. Cette opacité pour l'acteur s'explique par «l'absorption de [l']attention dans le contenu, l'objet de [l']activité » (Petitmengin, 2010, p. 165). L'entretien d'explicitation (Vermersch, 1994) vise le passage d'une conscience préréfléchie à une conscience réfléchie de l'expérience par un acte de ré-flexion (flexion de la conscience sur elle-même) qui peut s'effectuer dans un temps hors de l'action. En effet, tant 
qu'elle se développe dans la présence à la situation, la conscience préréfléchie ne peut être atteinte en «temps réel » (Petitmengin, 2006), c'est-à-dire dans le temps de l'acte. La raison principale repose sur l'impossibilité d'orienter simultanément notre attention sur le « quoi » et le «comment», sur l'objet du processus et la manière dont nous la réalisons (Petitmengin, 2006). Cette exigence a conduit à une généralisation de méthodes de verbalisation de l'action, fondées sur l'organisation d'entretiens hors des temps de séquences globales d'activités professionnelles.

${ }^{9}$ La lune est une habileté spécifique de l'agrès saut qui consiste après une course d'élan à impulser sur un tremplin pour effectuer un renversement de 360 degrés avec un passage en appui sur les mains sur une table de saut. Le préappel correspond à la phase de transition entre la course et l'impulsion.

${ }^{10}$ Une sortie est une habileté qui clôture l'enchaînement gymnique; elle débute sur l'agrès pour se terminer sur les tapis de réception.

${ }^{11}$ Contrairement à la posture d'évocation de l'entretien d'explicitation (Vermersch, 1994), certains dispositifs méthodologiques encouragent "l'échange intersubjectif [qui] met en mouvement l'activité existante» (Clot, 2000, p. 146), qui ne vise pas une restitution de l'expérience sur le mode du "revécu», mais en produit une autre, actualisée dans l'espace dialogique. Toutefois, cette mise en mouvement s'inscrit généralement dans un processus de formation qui dépasse la finalité épistémique.

\section{Références}

Affergan, F. (1987). Exotisme et altérité. Essai sur les fondements d'une critique de l'anthropologie. Paris : Presses universitaires de France.

Agier, M. (2004). La sagesse de l'ethnologue. Paris : L'œil neuf.

Althabe, G. (1990). Ethnologie du contemporain et enquête de terrain. Terrain, 14, 126-131.

Althabe, G., \& Hernandez, V. A. (2004). Implication et réflexivité en anthropologie. Journal des anthropologues, 98-99, 15-36.

Amiel, P. (2004). Ethnométhodologie appliquée. Éléments de sociologie praxéologique. Paris : Presses du Lema.

Anadón, M., \& Guillemette, F. (2007). La recherche qualitative est-elle nécessairement inductive? Recherches qualitatives, Hors-série, 5, 26-37.

Arshad, M. (2000). Le «dire» du silence: Les ponctuations émotionnelles et cognitives des interactions. Communication et organisation, 18. Repéré à $\mathrm{http}: / /$ communicationorganisation.revues.org/2413

Beaud, S., \& Weber, F. (2003). Guide de l'enquête de terrain. Produire et analyser des données ethnographiques. Paris: La Découverte. 
Becker, H. (2002). Les ficelles du métier. Comment conduire sa recherche en sciences sociales. Paris : La Découverte.

Biache, M. J. (2002). Anthropologie cognitive des pratiques corporelles: Considérations épistémologiques (Habilitation à diriger la recherche inédite). Université Blaise Pascal, Clermont-Ferrand.

Bordeleau, L. P. (2005). Quelle phénoménologie pour quels phénomènes? Recherches qualitatives, 25(1), 103-127.

Bourdieu, P. (1993). La misère du monde. Paris : Seuil.

Caratini, S. (2004). Les non-dits de l'anthropologie. Paris : Presses universitaires de France.

Cefaï, D. (2010). L'engagement ethnographique. Paris : Éditions de l'École des hautes études en sciences sociales.

Clot, Y. (2000). La formation par l'analyse du travail: Pour une $3^{\mathrm{e}}$ voie. Dans B. Maggi (Éd.), Manières de penser, manières d'agir en éducation et en formation (pp. 133-156). Paris : Presses universitaires de France.

Cohen, P. (2002). Le chercheur et son double. À propos d'une recherche sur le vécu des jeunes de la Réunion face au sida. Dans C. Ghasarian (Éd.), De l'ethnographie à l'anthropologie réflexive (pp. 73-89). Paris : Armand Colin

Copans, J. (1999). L'enquête ethnographique de terrain. Paris : Nathan.

Dosse, F. (1995). L'empire du sens. L'humanisation des sciences humaines. Paris : La Découverte.

Durand, M. (2002). Chronomètre et survêtement. Reflet de l'expérience quotidienne d'enseignants en éducation physique. Paris : Éditions Revue EPS.

Durand, M., Hauw, D., Leblanc, S., Saury, J., \& Sève, C. (2005). Analyse de l'activité et entraînement en sport de haut niveau. Éducation permanente, 161, 54-68.

Filliettaz, L. (2011). Discours de formateurs et rapport au savoir : L'explicitation située de l'action comme discours professionnel. Lidil, 43, 11-25.

Geertz, C. (1973). The interpretation of cultures. New York, NY: Basic Books.

Hert, P. (2014). Le corps du savoir: Qualifier le savoir incarné du terrain. Études de communication, 42, 29-46.

Kant, E. (1964). Anthropologie d'un point de vue pragmatique (trad. M. Foucault). Paris : Vrin. (Ouvrage original publié en 1797).

Kilani, M. (1992). Introduction à l'anthropologie. Lausanne : Payot.

Kilani, M. (1995). L'invention de l'autre. Essais sur le discours anthropologique. Lausanne : Payot. 
Laplantine, F. (1987). Clefs pour l'anthropologie. Paris : Seghers.

Latour, B., \& Woolgar, S. (1988). La vie de laboratoire. La production des faits scientifiques. Paris : La Découverte.

Leservoisier, O. (2005). Terrains ethnographiques et hiérarchies sociales. Retour réflexif sur la situation d'enquête. Paris : Karthala.

Maturana, H. R., \& Varela, F. J. (1994). L'arbre de la connaissance: Racines biologiques de la compréhension humaine. Paris : Addison-Wesley.

Morissette, J., Demazière, D., \& Pépin, M. (2014). Vigilance ethnographique et réflexivité méthodologique. Recherches qualitatives, 33(1), 9-18.

Olivier de Sardan, J. P. (1995). La politique du terrain. Sur la production des données en anthropologie. Enquête, 1, Repéré à http://journals.openedition.org/enquete/263

Olivier de Sardan, J. P. (1996). La violence faite aux données. Enquête, 3, 31-59. Repéré à http://enquete.revues.org/363

Ouattara, F. (2004). Une étrange familiarité. Cahiers d'études africaines, 175, 635657. Repéré à http://étudesafricaines.revue.org/4765

Ouellet, P. (1992). Les changements de lieux. Culture et métaphore. Dans J. Mélançon (Éd.), Les métaphores de la culture (pp. 195-212). Laval : Presses de l'Université Laval.

Papinot, C. (2014). La relation d'enquête comme relation sociale. Épistémologie de la démarche de recherche ethnographique. Laval : Presses de l'Université Laval.

Passeron, J. C., \& Revel, J. (2005). Penser par cas. Paris : Éditions de l'École des hautes études en sciences sociales.

Pastré, P. (2011). La didactique professionnelle. Approche anthropologique $d u$ développement chez les adultes. Paris : Presses universitaires de France.

Peschard, I. (2004). La réalité sans représentation. La théorie énactive de la cognition et sa légitimité épistémologique (Thèse de doctorat inédite). École Polytechnique, Paris. Repéré à https://tel.archives-ouvertes.fr/tel-00007975/document

Petitmengin, C. (2006). Describing one's subjective experience in the second person. An interview method for the science of consciousness'. Phenomenology and the Cognitive Science, 5, 229-269.

Petitmengin, C. (2010). La dynamique pré-réfléchie de l'expérience vécue. Alter Revue de Phénoménologie, 18, 165-182.

Piard, C. (1992). Science et technique de l'éducation gymnique. Paris: Presses universitaires de France.

Polanyi, M. (1966). The tacit dimension. Chicago, Il: University of Chicago Press. 
Ricœur, P. (1985). Temps et récit. Tome III. Le temps raconté. Paris : Seuil.

Ricœur, P. (1990). Soi-même comme un autre. Paris : Seuil.

Rolland, C., \& Cizeron, M. (2015). Le raisonnement métaphorique comme ressort cognitif de l'activité experte des entraîneurs de gymnastique artistique. Recherches \& éducations, 13, 21-36.

Samurçay, R., \& Rabardel, P. (2004). Modèles pour l'analyse de l'activité et des compétences. Dans R. Samurçay, \& P. Pastré (Éds), Recherches en didactique professionnelle (pp. 163-180). Toulouse : Octarès.

Schön, D. A. (1994). Le praticien réflexif. À la recherche du savoir caché dans l'agir professionnel. Montréal : Logiques.

Steiner, P. (2008). Sciences cognitive, tournant pragmatique et horizons pragmatistes. Tracés, 15, 85-105.

Stroobants, M. (2009). Dénouer les ficelles du métier. Pour connecter les savoirs formels et informels. Techniques et culture, 51, 164-179.

Tochon, F. V. (1993). L'enseignant expert. Paris : Nathan.

Urbain, J. D. (2003). Ethnologue mais pas trop. Paris : Payot \& Rivages.

Varela, F. (1989). Autonomie et connaissance. Essai sur le vivant (trad. P. Bourgine, \& P. Dumouchel). Paris : Seuil.

Varela, F., Thompson, E., \& Rosch, E. (1993). L'inscription corporelle de l'esprit. Sciences cognitives et expérience humaine. Paris : Seuil.

Vermersch, P. (1994). L'entretien d'explicitation. Paris : ESF.

Vermersch, P. (1999). Pour une psychologie phénoménologique. Psychologie française, 44(1), 7-18.

Zeitler, A. (2003). Émergence de types et construction de forme signifiante pour l'action chez un enseignant débutant. Dans J. M. Barbier, \& M. Durand (Éds), L'analyse de l'activité. Approches situées. Recherche et formation (pp. 51-62). Paris : INRP.

Cathy Rolland est maittre de conférences, enseignante à l'UFR STAPS (Unité de formation et de recherche en sciences et techniques des activités physiques et sportives) et membre du laboratoire ACTé (Activité, connaissance, transmission, éducation - EA 4281) de l'Université Clermont Auvergne, à Clermont-Ferrand en France. Ses travaux de recherche portent sur l'analyse de l'activité des acteurs engagés dans des situations de formation (particulièrement dans les milieux d'éducation motrice et sportive), selon une perspective anthropophénoménologique et l'étude de la dynamique expérientielle de la connaissance.

Pour joindre l'auteure : Cathy.Rolland@uca.fr 